The Korean Journal of Parasitology

Vol. 42, No. 4. 169-174, December 2004

\title{
Organ-specific antigens of Clonorchis sinensis
}

\author{
Shunyu $\mathrm{LI}^{1), 2)}$, Byung-Suk $\mathrm{CHUNG}^{1)}$, Min-Ho $\mathrm{CHOI}^{1)}$ and Sung-Tae $\mathrm{HONG}^{1) *}$ \\ ${ }^{1)}$ Department of Parasitology and Tropical Medicine, Seoul National University College of Medicine and \\ Institute of Endemic Diseases, Seoul National University Medical Research Center, Seoul 110-799, Korea, \\ ${ }^{2)}$ Department of Parasitology, College of Medicine, Yanbian University, 133000 Yanji, Jilin Province, P.R. China
}

\begin{abstract}
This study was carried out to find out specific proteins from different organs of Clonorchis sinensis. Crude extract, organ-specific and excretory-secretory (ES) proteins were analyzed by immunoblot with infected human sera. The bands of 7- and 17-kDa were main component of intestinal fluid and ES protein and commonly found in all organspecific proteins. The 17-kDa protein was observed from ES antigen, intestinal fluid, eggs and sperms, 26- and 28$\mathrm{kDa}$ proteins were from the uterus, vitellaria, and ovary, and 34-, 37-, 43- and 50-kDa proteins were mainly from the testis and sperms. Serum of mice immunized with sperms reacted to the $50-\mathrm{kDa}$ protein by immunoblotting and immunohistochemical staining showed a positive reaction at the seminal receptacle and seminiferous tubule. The present results show that the 7-kDa protein is a common antigen of every part or organ of $C$. sinensis, but different organs express their specific antigenic protein bands.
\end{abstract}

Key words: Clonorchis sinensis, organ-specific antigen, immunoblot, 7-kDa antigen

\section{INTRODUCTION}

Clonorchis sinensis (Cobbold, 1907) is a liver fluke of human which lives in the bile duct. It is an important parasite of human in Eastern Asia, including China, Taiwan, northern Vietnam and Korea. In endemic areas, there are at least 15 million people infected with C. sinensis over the world (Hong, 2003). In Korea, it is well known that human infection of $C$. sinensis is widely distributed along the major rivers and streams (Rim, 1986) and the prevalence of C. sinensis infection was $1.4 \%$ over the country (MHW \& KAHP, 1997).

Although stool examination is the standard diag-

- Received 1 November 2004, accepted after revision 17 November 2004.

- This study was supported by the research grant from the Korea Research Foundation (KRF-99-041-F00095), 1999-2001.

*Corresponding author (e-mail: hst@snu.ac.kr) nostic method for clonorchiasis, it is becoming difficult due to lack of compliance. In order to control clonorchiasis in endemic areas, effective mass screening is necessary; accordingly, serodiagnosis is now rapidly replacing the stool examination. Diagnosis by conventional ELISA, using the crude antigen of $C$. sinensis, is known to be sensitive and specific, but cross-reactions are known to occur (Choi et al., 2003b). The excretory-secretory (ES) protein of $C$. sinensis has been suggested to be a more sensitive and specific antigen than crude antigen for diagnosis of human clonorchiasis (Choi et al., 2003a).

Several studies reported that 7- , 8-, 26-, 28-, 34-, 37-, 43-, 70-, and 100-kDa bands are candidate antigenic proteins for serodiagnosis of clonorchiasis (Yong et al., 1991; Kim, 1994; Hong et al., 1997; Choi et al., 2003b). Recently, a few recombinant proteins of $C$. sinensis were produced and characterized, i.e., phosphoglycerate kinase and 28-kDa glutathione S-trans- 
ferase were found mainly in the tegument (Hong et al., 2000; Kang et al., 2001). Moreover, purified 17-kDa protein was found in eggs and reproductive organs, and 21-kDa myoglobin of $C$. sinensis was found over the whole body, including the tegument, oral sucker and vitellaria (Chung et al., 2003; Sim et al., 2003).

In the present study, we prepared soluble antigens of ES protein, intestinal fluid, testis, sperm, vitellaria, and eggs of $C$. sinensis separately and compared their antigenic components to overview its antigenic proteins.

\section{MATERIALS AND METHODS}

\section{Collection of $C$. sinensis}

Metacercariae of $C$. sinensis were isolated from naturally infected freshwater fish, Pseudorasbora parva, caught at Shenyang, Liaoning Province, China. The fish were digested by artificial gastric juice and the metacercariae were collected as described previously (Choi et al., 2003b). The metacercariae were orally fed to rabbits and adult flukes were recovered from their liver 8 weeks after the infection. The flukes were harvested from the bile duct of the liver, and washed several times with cold phosphate buffered saline solution (PBS, $\mathrm{pH} 8.0$ ).

\section{Preparation of $C$. sinensis organ-specific anti- gens}

To prepare organ-specific antigens of $C$. sinensis, such as the testis, sperm, vitellaria, egg, and intestinal fluid, the adult flukes were dissected by individual organs. The testis, seminal receptacle, vitellaria, uterus and intestinal fluid were separated and collected. Whole flukes or the separated organs were homogenized and centrifuged at $12,000 \mathrm{rpm}$ for $1 \mathrm{hr}$ at $4^{\circ} \mathrm{C}$. The supernatant was used as crude or individual organ antigens. To prepare ES protein, 100 living $C$. sinensis adults were incubated in PBS containing penicillin G and streptomycin (Life Technologies; USA) at $37^{\circ} \mathrm{C}$ under $5 \% \mathrm{CO}_{2}$ for $15 \mathrm{hr}$. The antigens were stored at $-70^{\circ} \mathrm{C}$ until used.

\section{Sera of infected humans}

A total of 100 serum samples was collected from clonorchiasis patients at an endemic locality in Heilongjiang, China. All infected subjects had a history of consuming raw freshwater fish and were egg positive by stool examination. Control sera were obtained from 24 healthy students at the Seoul National University.

\section{SDS-PAGE and immunoblot assay}

SDS-PAGE was prepared and run as described elsewhere (Laemmli, 1970). The gels were stained with Coomassie R-250 and observed for the protein bands. After electrophoresis, the protein bands in the gels were transferred onto PVDF membrane (Millipore Co.) sheets as usual. The membrane was cut into strips and the strips were blocked with 3\% skim milk (Difco Lab). After washing with PBST, the strips were incubated in sera (1:100 dilution) for $2 \mathrm{hr}$, and then in the secondary antibody for $1 \mathrm{hr}$, peroxidase conjugated goat anti-human IgG (AB Chem, Cambridge). The color reaction developed with 4-chloro-1-naphtol and $\mathrm{H}_{2} \mathrm{O}_{2}$.

\section{Preparation of immune serum}

Five BALB/c mice, 4-week old, were immunized by intraperitoneal injection with $100 \mu \mathrm{g}$ sperm protein mixed in an equal volume of Freund's complete adjuvant. A boosting injection followed two weeks later with a mixture of the protein and incomplete adjuvant. After 2 weeks, the mice received another boosting intravenous injection of $25 \mu \mathrm{g}$ antigen and were bled 3 days after the final boosting. Their sera were collected and frozen at $-70^{\circ} \mathrm{C}$ until use.

\section{Immunohistochemical staining}

The livers of $C$. sinensis infected rabbits 12 weeks after infection were fixed in $10 \%$ neutral formalin and prepared by paraffin embedding for immunohistochemical staining. The immune serum was diluted to 1:100 with PBS and preimmune BALB/c mouse serum was used for negative control. The sections were reacted with the sera for $3 \mathrm{hr}$ and washed twice with the buffer and incubated with biotinylated anti-mouse 


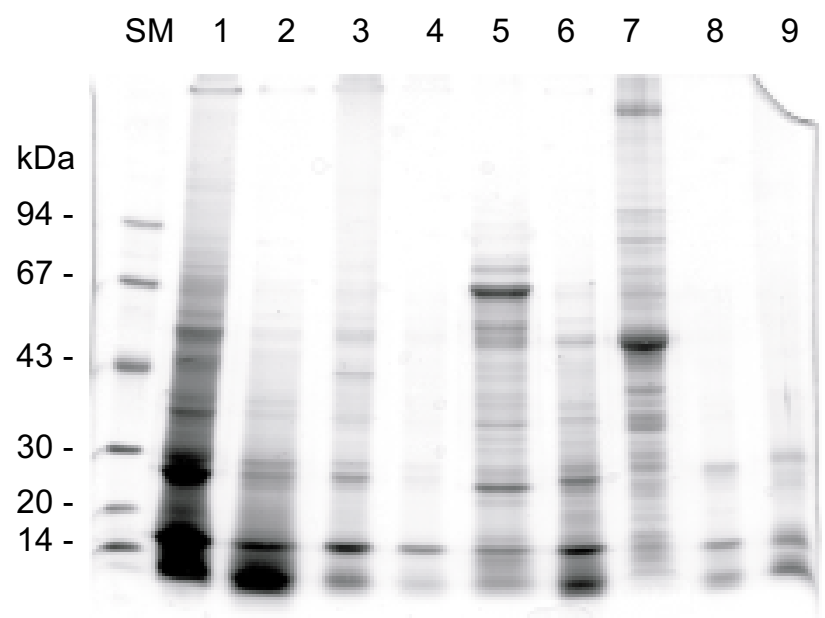

Fig. 1. SDS-PAGE analysis of soluble proteins extracted from several organs of Clonorchis sinensis. Lane 1, crude antigen; lane 2, vitellaria; lane 3, uterus; lane 4, ovary; lane 5, egg; lane 6, testis; lane 7, sperm; lane 8 , excretory-secretory proteins; lane 9 , intestinal fluid.

antibody at 1: 2000 dilution and in streptavidin-peroxidase conjugate solution for $20 \mathrm{~min}$. Color reaction developed in a substrate, 3-amino-9-ethylcarbazol (AEC), and counterstained with hematoxylin.

\section{RUSULTS}

\section{SDS-PAGE}

SDS-PAGE showed many protein bands ranging from 7 to $100 \mathrm{kDa}$ of relative molecular mass. Among them, 7 - and $17-\mathrm{kDa}$ proteins were commonly found in every organ of $C$. sinensis. Proteins with molecular masses of 26-, 28-, 34-, 37-, 47- and 55-kDa were found in female reproductive organs (uterus, vitellaria, ovary and eggs), and 34-, 37- and 50-kDa bands appeared in male reproductive organs (testis and sperms). Moreover, 7-, 17- and 28-kDa bands were observed in the ES protein and intestinal fluid protein (Fig. 1).

\section{Immunoblot patterns with IgG antibodies}

Immunoblotting with IgG antibodies of all egg positive cases reacted to 7-, 17-, 26-, 28-, 34-, 37-, 40-, 43-, 50-, 70- and 100-kDa bands. Among them, the 7-, 17-, 34- and $37-\mathrm{kDa}$ bands were found in the ES antigen, and 7- and 17-kDa bands were found in the intestinal fluid antigen. In a different running condition of PAGE, however, the 7-kDa band protein was detected in all organ-specific antigens. The $70-$ and $100-\mathrm{kDa}$ bands were detected only in crude antigen. The 7-, 26-, 28- and 34-kDa bands were observed in antigens of vitellaria, uterus and ovary, and the $10-\mathrm{kDa}$ band was observed in the egg antigen. The testis antigen showed 28-, 34- and 37-kDa bands and the sperm antigen showed 17-, 28-, 37- and 50-kDa bands (Fig. 2).

\section{Antigenicity of the sperm protein}

The immune serum from BALB/c mice immunized with the sperm antigen was ascertained by immunoblotting to react specifically to the $50-\mathrm{kDa}$

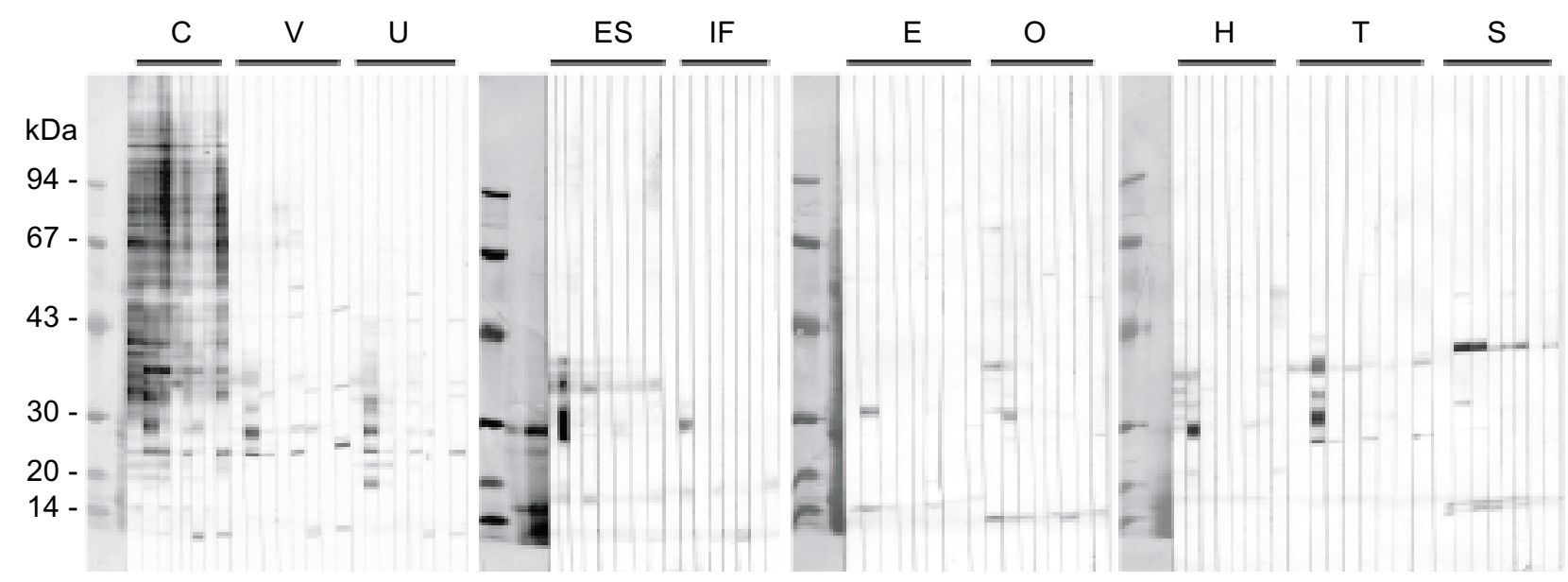

Fig. 2. Immunoblot patterns of anti-human IgG antibody to several antigens of Clonorchis sinensis. $\mathrm{C}$, crude extract; $\mathrm{V}$, vitellaria; U, uterus; ES, excretory-secretory protein; IF, intestinal fluid; E, eggs; O, ovary; H, head; T, testis; S, sperms. 


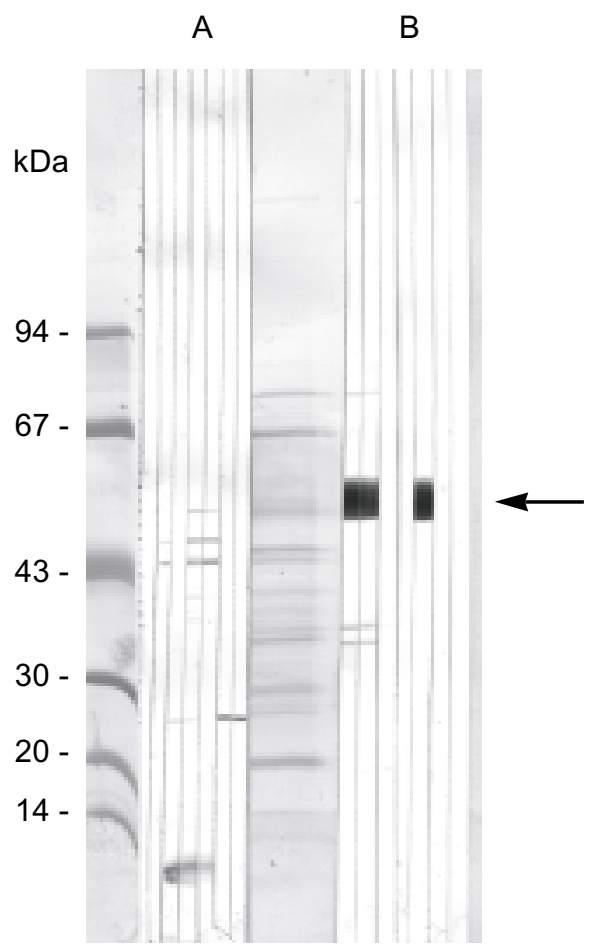

Fig. 3. Immunoblot of immunized serum with the sperm protein of Clonorchis sinensis. A, normal mouse serum; B, immunized mouse serum (arrow, 50-kDa band).

protein band (Fig. 3).

\section{Immunohistochemical staining}

Immunohistochemical staining using the immune serum showed that the protein was only distributed at the seminal receptacle and the seminiferous tubles of C. sinensis (Fig. 4).

\section{DISCUSSION}

The present study demonstrated numerous protein bands of $C$. sinensis. Of the proteins, immunoblotting with sera of clonorchiasis patients visualized 7-, 17-, 26-, 28-, 34-, 37-, 40-, 43-, 50-, 70- and 100-kDa antigenic bands. The whole pattern of antigenic bands is generally same as previously described (Hong et al., 1997; Choi et al., 2003b). However, the individual organs showed different patterns of antigen proteins.

The intestinal fluid consists of mainly 7- and 17-kDa proteins but the ES protein has two additional bands of 34- and $37-\mathrm{kDa}$. That means the intestinal fluid is a major component of the ES protein. In other words, the ES protein has other sources than the intestinal fluid, especially for the 34- and 37-kDa bands. The other sources may include semen fluid, uterine fluid, excretory bladder fluid or the tegument. Since the ES protein was found as a good serodiagnostic antigen to improve specificity, it is an important target of further study (Choi et al., 2003a).

The testis, seminal receptacle, seminal vesicle and seminiferous tubule are male reproductive organs. The seminal receptacle has been regarded as one of female organs because it is directly connected to the ovary, but it is full of sperms. Thus, it is included in male reproductive organs in the present study. It was easy to isolate the seminal receptacle and to collect pure sperms. The sperm protein showed 17-, 37- and $50-\mathrm{kDa}$ antigenic bands while the testis showed 34$\mathrm{kDa}$ band. Moreover, the testis was found very weakly antigenic. However, those bands are major antigenic components of $C$. sinensis which cross-reacted with sera of opisthorchiasis (Choi et al., 2003b). Sperms are produced in the testis, matured in the seminal vesicle, and excreted out through the genital pore. The sperms may be mixed with semen fluid to be excreted, and the proteins in the semen fluid may stimulate host immune system.

The female reproductive organs include the uterus, vitellaria, ovary, and eggs. Most of egg proteins consist of proteins from the vitellaria, and most of proteins from the uterus may be egg proteins. That is the reason why the female reproductive organs share most of the protein bands; 7-, 26-, 28, and 34-kDa. One egg protein of $28-\mathrm{kDa}$ was characterized from $C$. sinensis and found as a universal yolk protein of trematodes (Lee et al., 2004). Due to technical limitation, the seminal vesicle and seminiferous tubule were mixed in the tissue preparation of the uterus in the present study, which must be considered to explain the antigenic proteins of the uterus.

The 7-kDa protein was commonly found in all organ-specific antigens as well as in the ES antigen or intestinal fluid antigen. The 7-kDa band was actually a mixture of 7- and 8-kDa bands which were hardly differentiated by SDS-PAGE. The $8-\mathrm{kDa}$ band was 

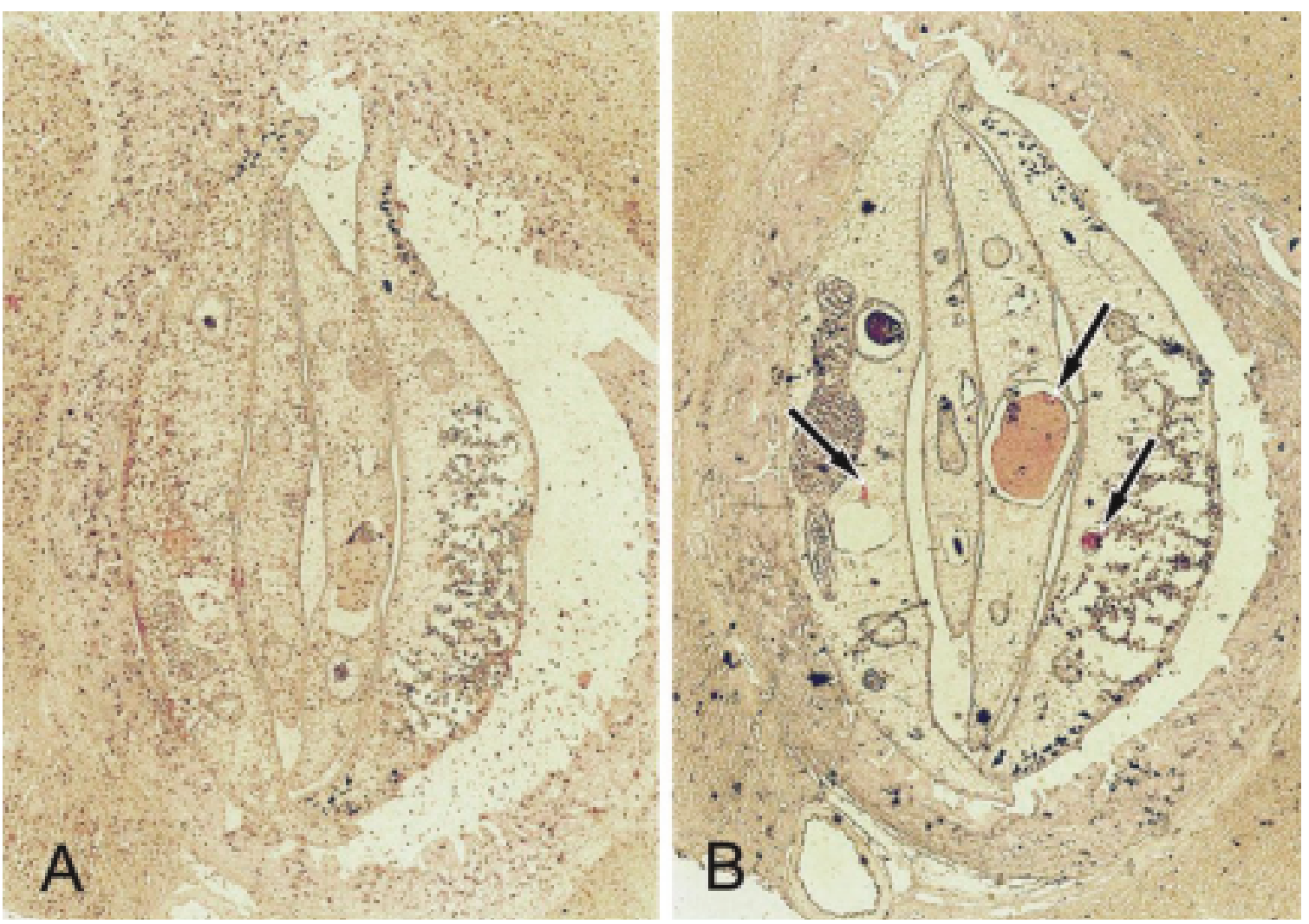

Fig. 4. Immunohistochemical staining of adult Clonorchis sinensis. A. Normal control serum shows no positive reaction. B. Staining with the immune serum shows positive staining in the seminal receptacle (center arrow) and the seminiferous tubule (arrows).

observed mainly in the tegument (Chung et al., 2002). That may be an important point because some tegumental tissues were contaminated during the organ preparation of the uterus and testis. The proteins of 7and $8-\mathrm{kDa}$ are produced at the tegument or the intestine.

The 17-kDa protein appeared from the ES antigen, intestinal fluid, eggs and sperms. One 17-kDa protein was identified as a cysteine proteinase and myoglobin of C. sinensis (Chung et al., 2000, 2003). The present 17 $\mathrm{kDa}$ protein is also a mixture of several different proteins from the intestinal fluid, eggs and sperms.

Two proteins of 26- and 28-kDa were observed from female reproductive organs and eggs. The 26and $28-\mathrm{kDa}$ proteins are known as glutathione $S$ transferase, which is a promising antigenic candidate (Kang et al., 2001).

Of the above mentioned antigenic proteins, 70- and $100-\mathrm{kDa}$ bands were detected only in crude antigen.
The two bands reacted with all of patients' sera. Also these bands are cross-reacting with sera of opisthorchiasis very frequently (Choi et al., 2003b). The two proteins are target antigens to reduce cross reaction with other trematodiasis for improved diagnostic specificity.

The immune serum from BALB/c mice revealed that the $50-\mathrm{kDa}$ protein is localized at the seminal receptacle and seminiferous tubule, but not at the testis. This result supported that the protein of $C$. sinensis sperms may be expressed in mature sperms or in semen fluid. This finding is consistent with that of F. hepatica (Stitt et al., 1992). The 50-kDa protein from the male reproductive organs may be a candidate molecule of anthelminthic medication or immunization.

In conclusion, the present results suggest that different antigenic proteins are produced by individual organs of $C$. sinensis. The specific antigenic proteins 
could be used as the diagnostic antigen for human clonorchiasis.

\section{REFERENCES}

Choi MH, Park IC, Li S, Hong ST (2003a) Excretory-secretory antigen is better than crude antigen for the serodiagnosis of clonorchiasis by ELISA. Korean J Parasitol 41: 3539.

Choi MH, Ryu JS, Lee M, et al. (2003b) Specific and common antigens of Clonorchis sinensis and Opisthorchis viverrini (Opisthorchidae, Trematoda). Korean J Parasitol 41: 155163.

Chung YB, Lee M, Yang HJ, et al. (2002) Characterization of partially purified $8 \mathrm{kDa}$ antigenic protein of Clonorchis sinensis. Korean J Parasitol 40: 83-88.

Chung YB, Yang HJ, Hong SJ, et al. (2003) Molecular cloning and immunolocalization of the $17 \mathrm{kDa}$ myoglobin of Clonorchis sinensis. Parasitol Res 90: 365-368.

Hong SJ, Kim TY, Song KY, Sohn WM, Kang SY (2001) Antigenic profile and localization of Clonorchis sinensis proteins in the course of infection. Korean J Parasitol 39: 307-312.

Hong SJ, Seong KY, Sohn WM, Song KY (2000) Molecular cloning and immunological characterization of phosphoglycerate kinase from Clonorchis sinensis. Mol Biochem Parasitol 108: 207-216.

Hong ST (2003) Clonorchis sinensis. In International Handbook of Foodborne Pathogens. pp 581-592. Marcel \& Dekker, New York, USA.

Hong ST, Kho WG, Lee M, Lee JS, Lee SH (1997) Immunoblot patterns of clonorchiasis. Korean J Parasitol 35: 87-93.
Kang SY, Ahn IY, Park CY, et al. (2001) Clonorchis sinensis: Molecular cloning and characterization of $28-\mathrm{kDa}$ glutathione S-transferase. Exp Parasitol 97: 186-195.

Kim SI (1994) Immune reactions between excretory-secretory antigens and specific antibodies of Clonorchis sinensis before and after praziquantel treatment in experimentally infected rabbits. Korean J Parasitol 32: 35-42.

Kim SI (1998) A Clonorchis sinensis-specific antigen that detects active human clonorchiasis. Korean J Parasitol 36: 37-45.

Laemmli UK (1970) Cleavage of structural proteins during assembly of the head of bacteriophage T4. Nature 227: 680-685.

Lee M, Chung YB, Lee SK, et al. (2004) The identification of a Clonorchis sinensis gene encoding an antigenic egg protein. Parasitol Res (in press).

Ministry of Health and Welfare, Korea Association of Health Promotion (1997) Prevalence of intestinal parasitic infection in Korea. The sixth report, Seoul, pp 20-48.

Rim HJ (1986) The current pathobiology and chemotherapy of clonorchiasis. Korean J Parasitol 24 (suppl): 1-141.

Sim S, Park GM, Yong TS (2003) Cloning and characterization of Clonorchis sinensis myoglobin using immune sera against excretory-secretory antigens. Parasitol Res 91: 338-343.

Stitt AW, Fairweather I, Trudgett AG, Johnston CF (1992) Fasciola hepatica: localization and partial characterization of tubulin. Parasitol Res 78: 103-107.

Yong TS, Im K, Chung PR (1991) Analysis of Clonorchis sinensis antigens and diagnosis of clonorchiasis using monoclonal antibodies. Korean J Parasitol 29: 293-310. 